\title{
Hyperpigmentation in Laugier-Hunziker syndrome
}

\author{
Dong-Lai Ma MD, Sergio Vano-Galvan MD PhD
}

Competing interests: None declared.

This article has been peer reviewed.

Affiliations: From the Department of Dermatology (Ma), Peking Union Medical College Hospital,

Chinese Academy of Medical Sciences and Peking Union Medical College, Beijing, China; and the Department of Dermatology (VanoGalvan), Hospital Ramon y Cajal, Madrid, Spain

\section{Correspondence to:} Dr. Dong-Lai Ma, mdonglai@public3.bta.net.cn

CMAJ 2011. DOI:10.1503 /cmaj.110211

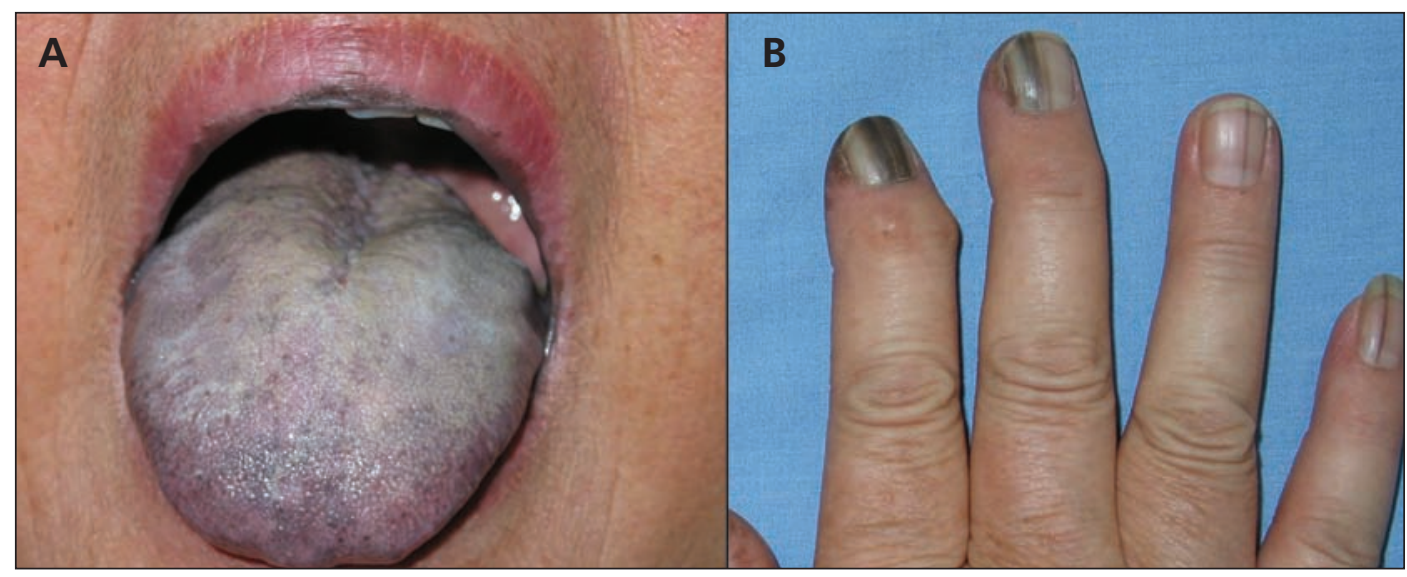

Figure 1: (A) Tongue of a 60-year-old woman showing widespread, diffuse pigmentation. (B) A striking pigmentary change over her fingernails appearing as discrete longitudinal bands of varying width.

A 60-year-old nonsmoking Chinese woman presented with a 40 -year history of progressive blackish pigmentation of her tongue, oral mucosa and lips (Figure 1A; Appendix 1, available at www.cmaj.ca/lookup /supp1/doi:10.1503/cmaj.110211/-/DC1). In addition, she had a 10-year history of longitudinal pigmented bands on parts of her fingernails (Figure 1B). There was no relevant drug history, and no other family members were affected. Histology of the lesions of the lower lip showed a substantial increase of melanin in the basal keratinocytes but no increase in the number of melanocytes. Serum cortisol and adrenocorticotropic hormone levels were within normal limits. The patient underwent an upper gastrointestinal study, barium enema and colonoscopy, which showed no evidence of polyps. A diagnosis of Laugier-Hunziker syndrome was made. In follow-up over the next five years, an increasing number of pigmented macules was noted.

Mucocutaneous hyperpigmentation alone is not uncommon in Asian, black and other darkskinned people. ${ }^{1}$ Laugier-Hunziker syndrome, however, is an acquired pigmentary disorder of the lips, oral mucosa and fingers, and is known to be an entirely benign disease with no systemic manifestations. Although rarely reported in the literature, its incidence is unknown. It is likely underreported. Peutz-Jeghers syndrome, an autosomal dominant genetic disorder, shares some clinical features with this condition, but the pigmented macules in Peutz-Jeghers syndrome most often appear in early life and tend to fade with time, whereas the lesions of Laugier-Hunziker syndrome are progressively acquired after puberty. ${ }^{2}$ The presence of pigmentation of the nails is characteristic of Laugier-Hunziker syndrome and is uncommon in Peutz-Jeghers syndrome. Unlike Laugier-Hunziker syndrome, Peutz-Jeghers syndrome often involves a family history and an association with hamartomatous polyps of the bowel. Most importantly, patients with Laugier-Hunziker syndrome do not require monitoring for the development of malignancies, in contrast to those with Peutz-Jeghers syndrome, who have an increased risk of cancer. ${ }^{3}$

\section{References}

1. Siponen M, Salo T. Idiopathic lenticular mucocutaneous pigmentation (Laugier-Hunziker syndrome): a report of a case. Oral Surg Oral Med Oral Pathol Oral Radiol Endod 2003;96: 288-92.

2. Ayoub N, Barete S, Bouaziz JD, et al. Additional conjunctival and penile pigmentation in Laugier-Hunziker syndrome: a report of two cases. Int J Dermatol 2004;43:571-4.

3. Beggs AD, Latchford AR, Vasen HF, et al. Peutz-Jeghers syndrome: a systematic review and recommendations for management. Gut 2010;59:975-86. 